\title{
Multi-Objective Supply Chain Inventory Model with Demand Dependent Purchase Cost and Production Rate Dependent Production Cost
}

\author{
Satya Kumar Das \\ Department of Mathematics \\ Govt. General Degree College at Gopiballavpur-II, India \\ Email: satyakrdasmath75@gmail.com \\ Sahidul Islam \\ Department of Mathematics \\ University of Kalyani, India \\ Email: sahidul.math@gmail.com
}

\begin{abstract}
In this paper, we have developed an integrated production inventory model for a two echelon supply chain consisting of one manufacturer and one retailer. Purchase cost for the manufacturer is dependent on inventory lot size; production cost of the manufacturer is dependent on production rate. Also purchase cost of the retailer is dependent on demand rate of the customer. The proposed multi objective supply chain inventory model has been solved by various techniques like as Fuzzy programming technique with hyperbolic membership function (FPTHMF), Fuzzy non-linear programming technique (FNLP) and Fuzzy additive goal programming technique (FAGP), weighted Fuzzy non-linear programming technique (WFNLP) and weighted Fuzzy additive goal programming technique (WFAGP). A numerical example is provided to illustrate the proposed model. Finally sensitivity analysis and graphical representation have been shown.
\end{abstract}

Keywords: Inventory, Supply Chain, Multi-item, Fuzzy Techniques

\section{Introduction}

The supply chain inventory model deal with decision that minimum the total average cost or maximum the total average profit. In that way to construct a real life mathematical supply chain inventory model on base on various assumptions and notations and approximation. Supply Chain management has taken a very important and critical role for any company in the increase globalization and competition in the business. The success of any supply chain system in any business depends on its level of collaboration and integration. In this paper, it is important to mention that a supply chain composed of two stages is considered a complex system in which one is manufacturer and other one is retailer.

The supply chain may be coordinated in numerous approaches. Zhou and Li (2007) showed that the coordination among parties in the ordering method will increase the expected profit for the retailer as well as for the complete supply chain. A three layer multiple item production inventory model for multiple suppliers and retailers was formulated by Pal et. al (2012). Rau et al., (2003) studied on integrated inventory model for deteriorating items under a multi- echelon supply chain environment. Yang et al., (2000) discussed on economic ordering policy of deteriorated item for vendor and buyer: an integrated approach. Pal et al., (2014) formulated on a multiple echelon production 
inventory system with supply disruption. Pal et al. (2012) discussed three layer supply chain: a production inventory model for re-workable items. Goyal, (1976) discussed an integrated inventory model for a single supplier-single customer problem. Tzafestas and Kapsiotis (1994) coordinated control of manufacturing supply chain system using multilevel techniques. Cardenas-Barron and Sana (2014) studied on a production inventory model for a two echelon supply chain when demand is dependent on sales teams' initiatives.

In the real life situations, purchase cost or production cost depend on number of quantities, demand rate, selling price etc. Bhunia and Shaikh (2014) developed on a deterministic inventory model for deteriorating items with selling price dependent demand and three-parameter Weibull distributed deterioration. Sridevi et al (2010) studied on inventory model for deteriorating items with Weibull rate of replenishment and selling price dependent demand. Shah et al., (2009) discussed on lot size inventory model for the Weibull distributed deterioration rate with discounted selling price and stock-dependent demand. Alfares and Ghaithan (2016) considered an inventory and pricing model with price-dependent demand, time-varying holding cost, and quantity discounts multi item is also important in the inventory management system. Ghosh et al., (2015) presented a multi-item inventory model for deteriorating items in limited storage space with stock-dependent demand. Sadeghi (2015) discussed a multi-item integrated inventory model with different replenishment frequencies of retailers in a two-echelon supply chain management: a tuned-parameters hybrid meta-heuristic. Kotb and Fergancy (2011) developed multi-item EOQ model with both demand depended unit cost and varying lead time via geometric programming. Cárdenas-Barrón and Sana (2015) considered multi-item EOQ inventory model in a two-layer supply chain while demand varies with promotional effort.

The concept of fuzzy set theory was first introduced by Zadeh (1965). Afterward Zimmermann (1985) applied the fuzzy set theory concept with some useful membership functions to solve the linear programming problem with some objective functions. Roy and Maiti (1998) considered multi-objective inventory models of deteriorating items with some constraints in a fuzzy environment. Wee et al., (2009) studied on multi-objective joint replenishment inventory model of deteriorated items in a fuzzy environment. Mandal and Islam (2016) formulated a fuzzy inventory model for deteriorating items with time depended demand and shortages under fully backlogged condition.

In this article, an integrated production inventory model for a two echelon supply chain has been considered in which purchase cost for the manufacturer is dependent on inventory lot size, production cost of the manufacturer is dependent on production rate and Purchase cost of the retailer is dependent on demand rate of the customer. Idle time cost and multi-item have been considered in this supply chain inventory model. Average cost is calculated in manufacturer individual inventory level as well as retailer individual inventory level and these are adding in integrated inventory level. The proposed MOSCIM has been solved by various techniques which are 
FPTHMF, FNLP, FAGP, WFNLP and WFAGP. A numerical example has been given to illustrate the paper. Sensitivity analysis and graphical representation have been shown. Finally conclusions have been drawn.

\section{Notations}

$I_{m r i}(t)$ :Manufacturer raw material inventory level of the $\mathrm{i}^{\text {th }}$ item at time $t$.

$I_{m f i}(t)$ : Manufacturer finished product level of the $\mathrm{i}^{\text {th }}$ item at time $t$.

$I_{r i}(t)$ : Retailer inventory level of the $\mathrm{i}^{\text {th }}$ item at time $t$.

$t_{i 1}$ : End of production time of the $\mathrm{i}^{\text {th }}$ item of the manufacturer.

$P_{i}$ : Production rate of the manufacturer of the $\mathrm{i}^{\text {th }}$ item.

$Q_{i}$ : The order quantity in the duration of a cycle of length for $\mathrm{i}^{\text {th }}$ item.

$h_{m r i}$ : Holding cost per unit per unit time of the raw material for $\mathrm{i}^{\text {th }}$ item.

$h_{m f i}$ : Holding cost per unit per unit time of the finished product for $\mathrm{i}^{\text {th }}$ item.

$h_{r i}$ : Holding cost per unit per unit time of the $\mathrm{i}^{\text {th }}$ item for retailer.

$A_{s i}$ : Set-up cost per order of $\mathrm{i}^{\text {th }}$ item for the manufacturer.

$A_{r i}$ : Set-up cost per order of $\mathrm{i}^{\text {th }}$ item for the retailer.

$I_{s i}:$ Cost per unit idle time of manufacturer.

$i_{r i}$ : Cost per unit idle time of retailer.

$T_{i}$ : The length of cycle time for $\mathrm{i}^{\text {th }}$ item.

$D_{i}$ : Customer demand rate per unit time for the $\mathrm{i}^{\text {th }}$ item.

$C_{P_{m}}^{i}$ : The unit purchase cost of the manufacturer of the $\mathrm{i}^{\text {th }}$ item.

$C_{P_{m}^{\prime}}^{i}$ : The unit production cost of the manufacturer of the $\mathrm{i}^{\text {th }}$ item.

$C_{P_{r}}^{i}$ : The unit purchase cost of the retailer of the $\mathrm{i}^{\text {th }}$ item.

$A C_{m}$ : Average cost of the manufacturer of the $\mathrm{i}^{\text {th }}$ item.

$A C_{r}$ : Average cost of the manufacturer of the $\mathrm{i}^{\text {th }}$ item.

$T A C_{i}(Q, P, D):$ Joint total average cost of the $\mathrm{i}^{\text {th }}$ item.

\section{Assumptions}

1. The multi item inventory system is developed.

2. The replenishment occurs instantaneously at infinite rate.

3. The lead time is neglected.

4. Shortages are not allowed.

5. Demand rate is constant.

6. The cost of idle times at manufacturer and retailer level are considered. 
7. The unit purchase cost $C_{P_{m}}^{i}$ of the $\mathrm{i}^{\text {th }}$ item of the manufacturer is inversely related to the lot size of the raw materials $Q_{i}$. So we take the following form $C_{P_{m}}^{i}\left(Q_{i}\right)=\delta_{i} Q_{i}^{-a_{i}}$ where $\delta_{i}>0$ and $a_{i}>0$ are constant real numbers.

8. The unit production cost $C_{P_{m}^{\prime}}^{i}$ of the $\mathrm{i}^{\text {th }}$ item of the manufacturer is considered as $C_{P_{m}^{\prime}}^{i}\left(P_{i}\right)=\frac{L_{i}}{P_{i}}+\sigma_{i} P_{i}$ where $L_{i}$ is the labor and energy costs of the $\mathrm{i}^{\text {th }}$ item which is equally distributed over the production rate. This cost is reduced by increases the production lot size. The second term $\sigma_{i} P_{i}$ represents tool/die costs which are proportional to the production rate.

9. The unit purchase cost $C_{P_{r}}^{i}$ of the retailer is inversely related to the demand rate $D_{i}$ of the customer. So we take the following form $C_{P_{r}}^{i}\left(D_{i}\right)=\mu_{i} D_{i}^{-b_{i}}$ where $\mu_{i}>0$ and $b_{i}>1$ are constant real numbers.

10. Holding cost of the raw materials, finished products of the supply chain is different. 11. Ratio of the raw material and finished good is $1: 1$

\section{Model formation in crisp for $i^{\text {th }}$ item}

\subsection{The manufacturer individual inventory model}

At this manufacturer inventory level (figure 1), the production starts with raw materials order lot size is $Q_{i}$. In this level, two situations occur: the first one is when the raw material decreases with production rate $P_{i}$ and it reaches to zero at time $t_{i 1}$. The second one is when the finished products, after production, pile up with rate $P_{i}$ up to time $t_{i 1}$, and it reaches to $Q_{i}$ at the time $t_{i 1}$. The governing differential equations are as following

$$
\frac{d I_{m r i}(t)}{d t}=-P_{i}, 0 \leq t \leq t_{i 1}
$$

With the boundary conditions, $I_{m r i}(0)=Q_{i}, I_{m r i}\left(t_{i 1}\right)=0$

And for finished products

$$
\frac{d I_{m f i}(t)}{d t}=P_{i}, 0 \leq t \leq t_{i 1}
$$

With the boundary conditions, $I_{m f i}(0)=0, I_{m f i}\left(t_{i 1}\right)=Q_{i}$

Solving the above differential equations (1) and (2) we get

$$
I_{m r i}(t)=Q_{i}-P_{i} t, \quad 0 \leq t \leq t_{i 1}
$$

and $I_{m f i}(t)=Q_{i}-P_{i}\left(t_{i 1}-t\right), 0 \leq t \leq t_{i 1}$ 
where $\quad t_{i 1}=\frac{Q_{i}}{P_{i}}$

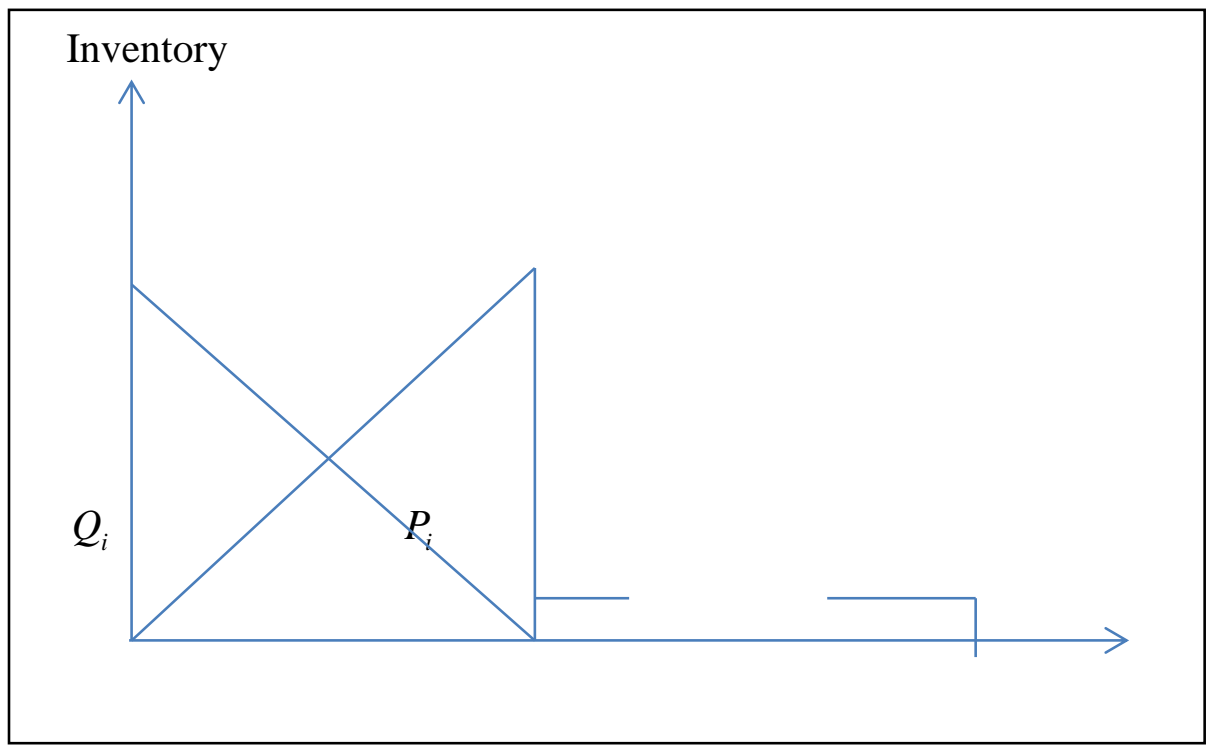

Figure 1: Inventory level for $\mathrm{i}^{\text {th }}$ item of the manufacture

Now we are calculating the various cost as following

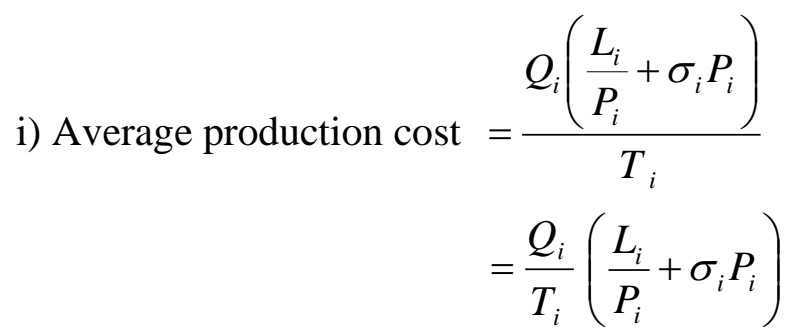

ii) Average purchased cost $=\frac{\delta_{i} Q_{i}^{1-a_{i}}}{T_{i}}$

iii) Average holding cost of raw materials $=\frac{h_{m r i}}{T_{i}} \int_{0}^{t_{i 1}} I_{m r i}(t) d t$

$$
=\frac{h_{m r i} Q_{i}^{2}}{2 T_{i} P_{i}}
$$

iv) Average holding cost of finished products $=\frac{h_{m f i}}{T_{i}} \int_{0}^{t_{i 1}} I_{m f i}(t) d t$

$$
=\frac{h_{m f i} Q_{i}^{2}}{2 T_{i} P_{i}}
$$

v) Average set-up-cost $=\frac{A_{s i}}{T_{i}}$ 
vi) Average idle time cost $=\frac{i_{s i}\left(T_{i}-t_{i 1}\right)}{T_{i}}$

Therefore average cost of the manufacturer is

$$
\left.A C_{m}=<\text { Average production cost }>+<\text { Average purchasing cost }\right\rangle+<\text { Average }
$$

holding cost of raw materials $>+<$ Average holding cost of finished products $>+<$ Average set-up-cost $>+<$ Average idle time cost $>$

$$
A C_{m}=\frac{\delta_{i} Q_{i}^{1-a_{i}}}{T_{i}}+\frac{Q_{i}}{T_{i}}\left(\frac{L_{i}}{P_{i}}+\sigma_{i} P_{i}\right)+\frac{h_{m r i} Q_{i}^{2}}{2 T_{i} P_{i}}+\frac{h_{m f i} Q_{i}^{2}}{2 T_{i} P_{i}}+\frac{A_{s i}}{T_{i}}+\frac{i_{s i}\left(T_{i}-t_{i 1}\right)}{T_{i}}
$$

Hence

$$
\begin{aligned}
& A C_{m}\left(Q_{i}, P_{i}, D_{i}\right)=\frac{\delta_{i} Q_{i}^{-a_{i}} P_{i} D_{i}}{\left(P_{i}+D_{i}\right)}+\frac{P_{i} D_{i}}{\left(P_{i}+D_{i}\right)}\left(\frac{L_{i}}{P_{i}}+\sigma_{i} P_{i}\right)+\frac{h_{m i} Q_{i} D_{i}}{2\left(P_{i}+D_{i}\right)}+\frac{h_{m f i} Q_{i} D_{i}}{2\left(P_{i}+D_{i}\right)}+ \\
& \frac{A_{s i} P_{i} D_{i}}{Q_{i}\left(P_{i}+D_{i}\right)}+\frac{i_{s i} P_{i}}{\left(P_{i}+D_{i}\right)}
\end{aligned}
$$

for $i=1,2,3, \ldots \ldots ., n$

\subsection{The retailer individual inventory model}

At this inventory level (figure 2), the corresponding differential equation is

$$
\frac{d I_{r i}(t)}{d t}=-D_{i}, \quad t_{i 1} \leq t \leq T_{i}
$$

With boundary conditions, $I_{r i}\left(t_{i 1}\right)=Q_{i}, I_{r i}\left(T_{i}\right)=0$

Solving the above differential equation (8) we get,

$$
\begin{aligned}
I_{r i}(t) & =Q_{i}-D_{i}\left(t-t_{i 1}\right), \quad t_{i 1} \leq t \leq T_{i} \\
\text { and } T_{i} & =\frac{Q_{i}}{D_{i}}+\frac{Q_{i}}{P_{i}}
\end{aligned}
$$

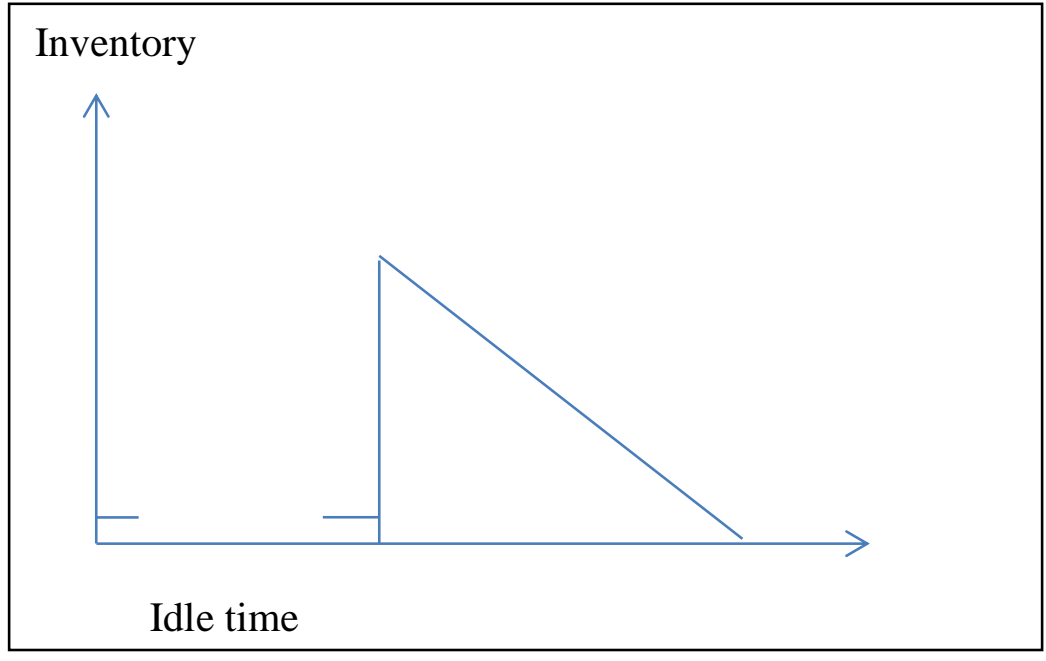


Figure 2: Inventory situation for $i^{\text {th }}$ item of the retailer

Now we calculate the various costs as following

i) Average purchased cost $=\frac{\mu_{i} D_{i}^{-b_{i}} Q_{i}}{T_{i}}$

ii) Average holding cost $=\frac{h_{r i}}{T_{i}} \int_{t_{i 1}}^{T_{i}} I_{r i}(t) d t$

$$
=\frac{h_{r i} Q_{i}^{2}}{2 T_{i} D_{i}}
$$

iii) Average set-up-cost $=\frac{A_{r i}}{T_{i}}$

iv) Average Idle time cost $=\frac{i_{r i} t_{i 1}}{T_{i}}$

Therefore the average cost for the retailer individual inventory level is

$A C_{r}=<$ Average purchased cost $>+<$ Average holding cost $>+<$ Average set-upcost $>+<$ Average Idle time cost $>$

$$
\begin{aligned}
& A C_{r}=\frac{\mu_{i} D_{i}^{-b_{i}} Q_{i}}{T_{i}}+\frac{h_{r i} Q_{i}^{2}}{2 T_{i} D_{i}}+\frac{A_{r i}}{T_{i}}+\frac{i_{r i} t_{i 1}}{T_{i}} \\
& A C_{r}\left(Q_{i}, P_{i}, D_{i}\right)=\frac{\mu_{i} D_{i}^{1-b_{i}} P_{i}}{\left(P_{i}+D_{i}\right)}+\frac{h_{r i} Q_{i} P_{i}}{2\left(P_{i}+D_{i}\right)}+\frac{A_{r i} P_{i} D_{i}}{\left(P_{i}+D_{i}\right) Q_{i}}+\frac{i_{r i} D_{i}}{\left(P_{i}+D_{i}\right)}
\end{aligned}
$$

\subsection{The integrated inventory model}

Therefore the total average cost for $\mathrm{i}^{\text {th }}$ item in integrated inventory model is

$T A C_{i}\left(Q_{i}, P_{i}, D_{i}\right)=A C_{m}\left(Q_{i}, P_{i}, D_{i}\right)+A C_{r}\left(Q_{i}, P_{i}, D_{i}\right)$

$$
\begin{gathered}
\operatorname{TAC}_{i}\left(Q_{i}, P_{i}, D_{i}\right)=\frac{\delta_{i} Q_{i}^{-a_{i}} P_{i} D_{i}}{\left(P_{i}+D_{i}\right)}+\frac{P_{i} D_{i}}{\left(P_{i}+D_{i}\right)}\left(\frac{L_{i}}{P_{i}}+\sigma_{i} P_{i}\right)+\frac{h_{m r i} Q_{i} D_{i}}{2\left(P_{i}+D_{i}\right)}+\frac{h_{m f i} Q_{i} D_{i}}{2\left(P_{i}+D_{i}\right)}+ \\
\frac{\mu_{i} D_{i}^{1-b_{i}} P_{i}}{\left(P_{i}+D_{i}\right)}+\frac{h_{r i} Q_{i} P_{i}}{2\left(P_{i}+D_{i}\right)}+\frac{A_{r i} P_{i} D_{i}}{\left(P_{i}+D_{i}\right) Q_{i}}+\frac{i_{r i} D_{i}}{\left(P_{i}+D_{i}\right)}+\frac{A_{s i} P_{i} D_{i}}{Q_{i}\left(P_{i}+D_{i}\right)}+\frac{i_{s i} P_{i}}{\left(P_{i}+D_{i}\right)} \\
D_{i}>0, P_{i}>0, Q_{i}>0 \text { for } i=1,2,3, \ldots \ldots, n
\end{gathered}
$$

Here total average cost per unit time for each item can be considered as one objective function. So our proposed multi objective supply chain inventory model (MOSCIM) can be written as

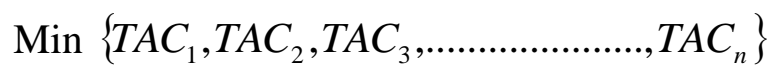

Subject to, $D_{i}>0, P_{i}>0, Q_{i}>0$ 
Where

$$
\begin{gathered}
T A C_{i}\left(Q_{i}, P_{i}, D_{i}\right)=\frac{\delta_{i} Q_{i}^{-a_{i}} P_{i} D_{i}}{\left(P_{i}+D_{i}\right)}+\frac{P_{i} D_{i}}{\left(P_{i}+D_{i}\right)}\left(\frac{L_{i}}{P_{i}}+\sigma_{i} P_{i}\right)+\frac{h_{m r i} Q_{i} D_{i}}{2\left(P_{i}+D_{i}\right)}+\frac{h_{m f i} Q_{i} D_{i}}{2\left(P_{i}+D_{i}\right)}+ \\
\frac{\mu_{i} D_{i}^{1-b_{i}} P_{i}}{\left(P_{i}+D_{i}\right)}+\frac{h_{r i} Q_{i} P_{i}}{2\left(P_{i}+D_{i}\right)}+\frac{A_{r i} P_{i} D_{i}}{\left(P_{i}+D_{i}\right) Q_{i}}+\frac{i_{r i} D_{i}}{\left(P_{i}+D_{i}\right)}+\frac{A_{s i} P_{i} D_{i}}{Q_{i}\left(P_{i}+D_{i}\right)}+\frac{i_{s i} P_{i}}{\left(P_{i}+D_{i}\right)}
\end{gathered}
$$

for $i=1,2,3, \ldots \ldots, n$

\section{Fuzzy programming technique with hyperbolic membership function (FPTHMF) for solving MOSCIM.}

Solve the MOSCIM (13) as a single objective NLP using only one objective at a time and we are ignoring the all other objectives. Repeat the process $n$ times for $n$ different objective functions. So we get the ideal solutions. Pay-off matrix is prepared by using the ideal solutions as follows:

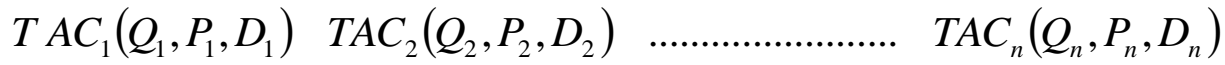

$$
\begin{aligned}
& \left(Q_{1}{ }^{1}, P_{1}{ }^{1}, D_{1}{ }^{1}\right)\left[T A C_{1}^{*}\left(Q_{1}{ }^{1}, P_{1}^{1}, D_{1}^{1}\right) \quad T A C_{2}\left(Q_{1}^{1}, P_{1}^{1}, D_{1}^{1}\right) \quad \ldots . \quad T A C_{n}\left(Q_{1}^{1}, P_{1}^{1}, D_{1}^{1}\right)\right. \\
& \left(Q_{2}^{2}, P_{2}^{2}, D_{2}^{2}\right) \quad T A C_{2}\left(Q_{2}^{2}, P_{2}^{2}, D_{2}^{2}\right) \quad T A C_{2}^{*}\left(Q_{2}^{2}, P_{2}^{2}, D_{2}^{2}\right) \quad \ldots . T A C_{n}\left(Q_{2}^{2}, P_{2}^{2}, D_{2}^{2}\right)
\end{aligned}
$$

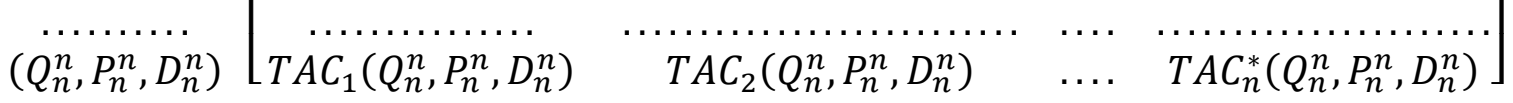

Let $U^{k}=\operatorname{Max}\left\{\operatorname{TAC}_{k}\left(Q_{i}^{i}, P_{i}^{i}, D_{i}^{i}\right), i=1,2, \ldots . ., n\right\}$ for $k=1,2,3, \ldots ., n$

and $L^{k}=T A C_{k}^{*}\left(Q_{k}^{k}, P_{k}^{k}, D_{k}^{k}\right)$ for $k=1,2,3, \ldots ., n$

Therefore $L^{k} \leq T A C_{k}\left(Q_{i}^{i}, P_{i}^{i}, D_{i}^{i}\right) \leq U^{k}$ for $i=1,2,3, \ldots ., n$ and $k=1,2,3, \ldots ., n$

The non-linear hyperbolic membership function $\mu_{T A C_{i}}^{H}\left(T A C_{i}\left(Q_{i}, P_{i}, D_{i}\right)\right)$ for the $\mathrm{i}^{\text {th }}$ objective function $T A C_{i}\left(Q_{i}, P_{i}, D_{i}\right)$ is defined as follows:

$$
\mu_{T A C_{i}}^{H}\left(T A C_{i}\left(Q_{i}, P_{i}, D_{i}\right)\right)=\frac{1}{2} \tanh \left(\left(\frac{U^{i}+L^{i}}{2}-T A C_{i}\left(Q_{i}, P_{i}, D_{i}\right)\right) \rho_{i}\right)+\frac{1}{2}
$$

where $\rho_{i}$ is a parameter, $\rho_{i}=\frac{3}{\left(U^{i}-L^{i}\right) / 2}=\frac{6}{U^{i}-L^{i}}$ for $i=1,2,3, \ldots ., n$

Using the above membership function, fuzzy non-linear programming problem is formulated as follows:

$\operatorname{Max} \lambda$

Subject to, $\frac{1}{2} \tanh \left(\left(\frac{U^{i}+L^{i}}{2}-\operatorname{TAC}_{i}\left(Q_{i}, P_{i}, D_{i}\right)\right) \rho_{i}\right)+\frac{1}{2} \geq \lambda, \lambda \geq 0$

After simplification the above non-linear programming problem (18) can be written as

$\operatorname{Max} y$ 
Subject to, $y+\rho_{i}\left(T A C_{i}\left(Q_{i}, P_{i}, D_{i}\right)\right) \leq \frac{U^{i}+L^{i}}{2} \rho_{i}, y \geq 0, D_{i}>0, P_{i}>0, Q_{i}>0$

for $i=1,2,3, \ldots ., n$

The programming problem (19) can be solved by suitable mathematical programming algorithm and we shall get the solution of the MOSCIM (13).

\section{Fuzzy programming technique (based on max-min and max-additive operators)}

In this technique for solving MOSCIM (13), first we have to make pay-off matrix which has been shown in the above (14) and then to find $U^{i}$ and $L^{i}$, shown in equation no. (15) and (16). In this technique, the membership function $\mu_{T A C_{i}}\left(T A C_{i}\left(Q_{i}, P_{i}, D_{i}\right)\right)$ for the $\mathrm{i}^{\text {th }}$ objective function $\operatorname{TAC}_{i}\left(Q_{i}, P_{i}, D_{i}\right)$ is defined as follows:

$$
\mu_{T A C_{i}}\left(\operatorname{TAC}_{i}\left(Q_{i}, P_{i}, D_{i}\right)\right)=\left\{\begin{array}{cl}
1 & \text { for } T A C_{i}\left(Q_{i}, P_{i}, D_{i}\right)<L^{i} \\
\frac{U^{i}-T A C_{i}\left(Q_{i}, P_{i}, D_{i}\right)}{U^{i}-L^{i}} & \text { for } L^{i} \leq T A C_{i}\left(Q_{i}, P_{i}, D_{i}\right) \leq U^{i} \\
0 & \text { for } T A C_{i}\left(Q_{i}, P_{i}, D_{i}\right)>U^{i}
\end{array}\right.
$$

for $i=1,2,3, \ldots, n$.

\subsection{Fuzzy non-linear programming technique (FNLP) based on max-min operator}

Using the above membership function (20), fuzzy non-linear programming problem based on max-min operator is formulated as follows:

$\operatorname{Max} \alpha$

Subject to, $T A C_{i}\left(Q_{i}, P_{i}, D_{i}\right)+\alpha^{\prime}\left(U^{i}-L^{i}\right) \leq U^{i}, 0 \leq \alpha^{\prime} \leq 1, D_{i}>0, P_{i}>0, Q_{i}>0$

for $i=1,2,3, \ldots ., n$

The programming problem (21) can be solved by suitable mathematical programming algorithm and we shall get the solution of the MOSCIM (13).

\subsection{Fuzzy additive goal programming technique (FAGP) based on max-additive operator}

In this technique, using membership function (20), the fuzzy additive goal programming problem based on max-additive operator is formulated as follows:

$\operatorname{Max} \sum_{i=1}^{n} \frac{U^{i}-T A C_{i}\left(Q_{i}, P_{i}, D_{i}\right)}{U^{i}-L^{i}}$

Subject to, $L^{i}-T_{A} C_{i}\left(Q_{i}, P_{i}, D_{i}\right) \leq 0, D_{i}>0, P_{i}>0, Q_{i}>0$ for $i=1,2,3, \ldots ., n$ (22)

The programming problem (22) can be solved by suitable mathematical programming algorithm and we shall get the solution of the MOSCIM (13). 
7. Weighted fuzzy programming technique (based on max-min and maxadditive operators)

\subsection{Weighted fuzzy non-linear programming technique (WFNLP) based on max-min operator}

For this technique we consider positive weight $\omega_{i}$ for each objective $T A C_{i}\left(Q_{i}, P_{i}, D_{i}\right)$. Using the above membership function (20), the WFNLP based on max-min operator is stated as follows:

$\operatorname{Max} \alpha$

Subject to, $\omega_{i} \mu_{T A C_{i}}\left(T A C_{i}\left(Q_{i}, P_{i}, D_{i}\right)\right) \geq \alpha, 0 \leq \alpha \leq 1, D_{i}>0, P_{i}>0, Q_{i}>0$

$$
\text { and } \sum_{i=1}^{n} \omega_{i}=1 \text {, for } i=1,2,3, \ldots ., n
$$

The programming problem (23) can be solved by suitable mathematical programming algorithm and we shall get the solution of the MOSCIM (13).

\subsection{Weighted fuzzy additive goal programming technique (WFAGP) based on max-additive operator (WFAGP)}

Again using the above membership function (20), the WFAGP based on max-additive operator is formulated as follows

$\operatorname{Max} \sum_{i=1}^{n} \omega_{i} \mu_{T A C_{i}}\left(T A C_{i}\left(Q_{i}, P_{i}, D_{i}\right)\right)$

Subject to, $0 \leq \mu_{T A C_{i}}\left(T A C_{i}\left(Q_{i}, P_{i}, D_{i}\right)\right) \leq 1, D_{i}>0, P_{i}>0, Q_{i}>0$

and $\sum_{i=1}^{n} \omega_{i}=1$, for $i=1,2,3, \ldots, n$

The programming problem (24) can be solved by suitable mathematical programming algorithm and we shall get the solution of the MOSCIM (13).

\section{Numerical Example}

Let us consider two items with parameters value in proper unit.

Table 1: Input imprecise data for shape parameters

\begin{tabular}{|c|c|c|c|c|c|c|c|c|c|c|c|c|c|}
\hline \multirow[t]{2}{*}{ Item } & \multicolumn{13}{|c|}{ Parameters } \\
\hline & $\delta$ & $a$ & $L$ & $\sigma$ & $\mu$ & $b$ & $h_{m r}$ & $h_{m f}$ & $h_{r}$ & $A_{S}$ & $i_{S}$ & $A_{r}$ & $i_{r}$ \\
\hline 1 & 100 & 5 & 500 & 0.5 & 200 & 10 & 1.5 & 3 & 5 & 200 & 4 & 300 & 6 \\
\hline II & 200 & 7 & 800 & 0.6 & 300 & 11 & 1.0 & 2 & 4 & 300 & 3 & 400 & 5 \\
\hline
\end{tabular}


Multi-Objective Supply Chain Inventory Model with Demand Dependent Purchase Cost and Production Rate ......

Table 2: Optimal solutions of MOSCIM using different methods

\begin{tabular}{|l|c|c|c|c|c|c|c|c|}
\hline Methods & $P_{1}{ }^{*}$ & $D_{1}{ }^{*}$ & $Q_{1}{ }^{*}$ & $T A C_{1}{ }^{*}$ & $P_{2}{ }^{*}$ & $D_{2}{ }^{*}$ & $Q_{2}{ }^{*}$ & $T A C_{2}{ }^{*}$ \\
\hline FPTHMF & 28.51 & 1.41 & 16.44 & 137.23 & 33.57 & 1.44 & 22.07 & 162.08 \\
\hline FNLP & 28.51 & 1.41 & 16.33 & 137.23 & 33.57 & 1.44 & 22.07 & 162.08 \\
\hline FAGP & 28.39 & 1.41 & 16.34 & 137.23 & 33.57 & 1.44 & 22.07 & 162.08 \\
\hline
\end{tabular}

Table 3: Optimal solution of MOSCIM using different weights by WFNLP method

\begin{tabular}{|l|c|c|c|c|c|c|c|c|}
\hline weights & $P_{1}{ }^{*}$ & $D_{1}{ }^{*}$ & $Q_{1}{ }^{*}$ & $T A C_{1}{ }^{*}$ & $P_{2}{ }^{*}$ & $D_{2}{ }^{*}$ & $Q_{2}{ }^{*}$ & $T A C_{2}{ }^{*}$ \\
\hline$w_{1}=0.4, w_{2}=0.6$ & 28.49 & 1.41 & 16.42 & 137.23 & 29.81 & 1.43 & 17.83 & 164.39 \\
\hline$w_{1}=0.5, w_{2}=0.5$ & 28.34 & 1.41 & 16.31 & 137.23 & 33.57 & 1.44 & 22.07 & 162.08 \\
\hline$w_{1}=0.6, w_{2}=0.4$ & 31.91 & 1.41 & 16.77 & 137.49 & 33.57 & 1.44 & 22.07 & 162.08 \\
\hline
\end{tabular}

Table 4: Optimal solution of MOSCIM using different weights by WFAGP method

\begin{tabular}{|l|c|c|c|c|c|c|c|c|}
\hline weights & $P_{1}{ }^{*}$ & $D_{1}{ }^{*}$ & $Q_{1}{ }^{*}$ & $T A C_{1}{ }^{*}$ & $P_{2}{ }^{*}$ & $D_{2}{ }^{*}$ & $Q_{2}{ }^{*}$ & $T A C_{2}{ }^{*}$ \\
\hline$w_{1}=0.4, w_{2}=0.6$ & 28.46 & 1.40 & 16.44 & 137.24 & 3.57 & 1.41 & 22.07 & 162.27 \\
\hline$w_{1}=0.5, w_{2}=0.5$ & 28.48 & 1.41 & 16.34 & 137.23 & 33.57 & 1.44 & 22.07 & 162.08 \\
\hline$w_{1}=0.6, w_{2}=0.4$ & 28.24 & 1.41 & 16.41 & 137.23 & 33.57 & 1.44 & 22.07 & 162.08 \\
\hline
\end{tabular}

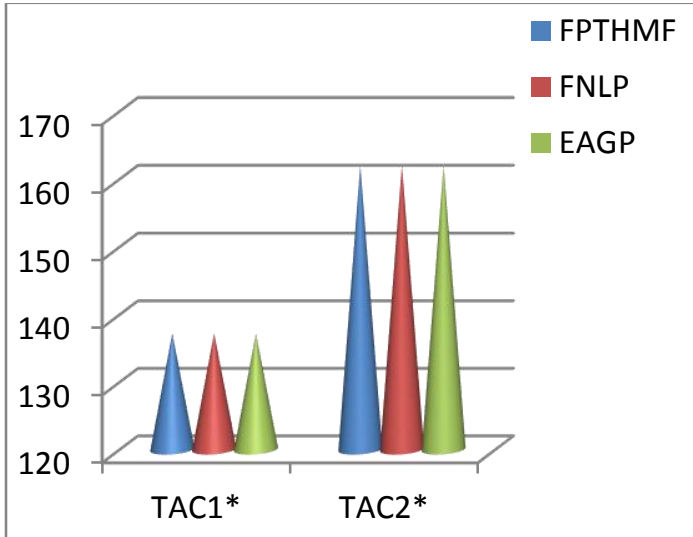

Figure 3: minimizing cost of $1^{\text {st }}$ and $2^{\text {nd }}$ items using different methods.

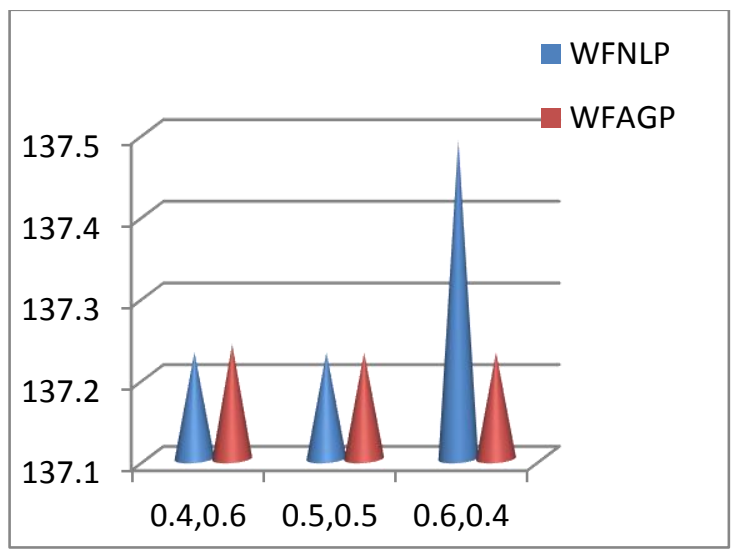

Figure 4: minimizing cost of $1^{\text {st }}$ item using different methods and different Weights.

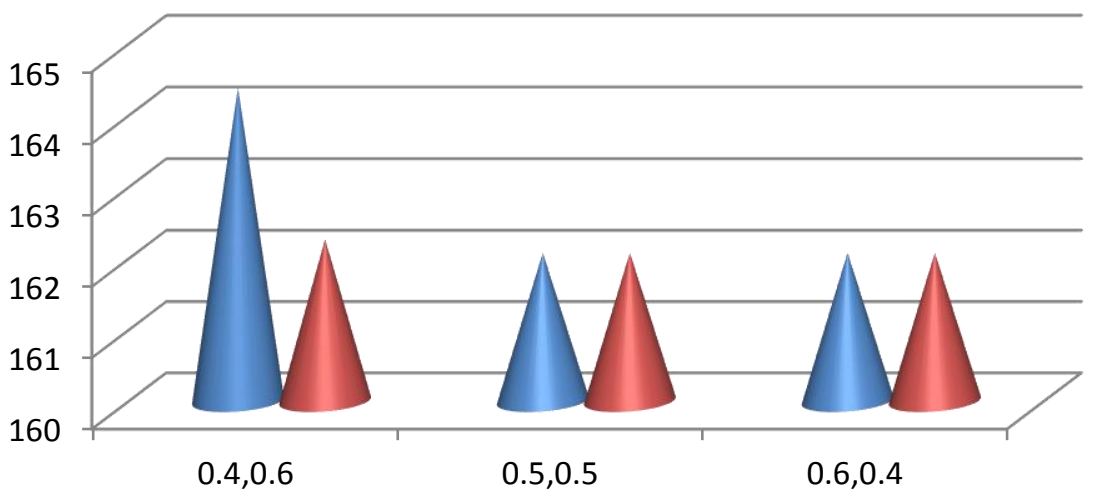

WFNLP

- WFAGP

Figure 5: minimizing cost of $2^{\text {nd }}$ item using different methods and different weights. 
From the above figure 3 shows that minimum costs of both items are same for all different methods. Figure 4 shows that minimum costs for $1^{\text {st }}$ item are almost same for different weights in WFNLP, WFAGP methods. Figure 5 shows that minimum costs for $2^{\text {nd }}$ item are almost same for different weights in WFNLP, WFAGP methods.

\section{Sensitivity Analysis}

Table 5: Optimal solution of MOSCIM by FPTHMF, FNLP and FAGP methods for different values of $L_{1}, L_{2}$.

\begin{tabular}{|l|c|c|c|c|c|c|c|c|c|c|}
\hline Method & $L_{1}$ & $L_{2}$ & $Q_{1}{ }^{*}$ & $P_{1}{ }^{*}$ & $D_{1}{ }^{*}$ & $T A C_{1}{ }^{*}$ & $Q_{2}{ }^{*}$ & $P_{2}{ }^{*}$ & $D_{2}{ }^{*}$ & $T A C_{2}{ }^{*}$ \\
\hline \multirow{5}{*}{ FPTHMF } & 500 & 800 & 16.44 & 28.51 & 1.41 & 137.23 & 22.07 & 33.57 & 1.44 & 162.08 \\
\cline { 2 - 11 } & 600 & 900 & 16.41 & 31.66 & 1.40 & 141.69 & 22.02 & 35.87 & 1.43 & 166.05 \\
\cline { 2 - 11 } & 700 & 1000 & 16.35 & 34.97 & 1.39 & 145.74 & 22.04 & 38.04 & 1.43 & 169.77 \\
\cline { 2 - 10 } & 800 & 1100 & 16.36 & 37.26 & 1.39 & 149.47 & 22.02 & 40.10 & 1.42 & 173.29 \\
\hline \multirow{5}{*}{ FNLP } & 500 & 800 & 16.34 & 28.51 & 1.41 & 137.23 & 22.07 & 33.57 & 1.44 & 162.08 \\
\cline { 2 - 10 } & 600 & 900 & 16.41 & 31.66 & 1.40 & 141.69 & 22.05 & 35.89 & 1.43 & 166.05 \\
\cline { 2 - 10 } & 700 & 1000 & 16.49 & 34.54 & 1.40 & 145.74 & 22.04 & 38.04 & 1.43 & 169.77 \\
\cline { 2 - 10 } & 800 & 1100 & 16.41 & 37.64 & 1.39 & 149.47 & 22.10 & 39.83 & 1.42 & 173.29 \\
\hline \multirow{5}{*}{ FAGP } & 500 & 800 & 16.34 & 28.39 & 1.41 & 137.23 & 22.07 & 33.57 & 1.44 & 162.08 \\
\cline { 2 - 10 } & 600 & 900 & 16.42 & 31.92 & 1.40 & 141.69 & 21.94 & 36.02 & 1.43 & 166.05 \\
\cline { 2 - 10 } & 700 & 1000 & 16.54 & 34.47 & 1.39 & 145.74 & 22.04 & 38.04 & 1.43 & 169.77 \\
\cline { 2 - 9 } & 800 & 1100 & 16.28 & 37.35 & 1.39 & 149.47 & 21.74 & 40.20 & 1.42 & 173.29 \\
\hline
\end{tabular}

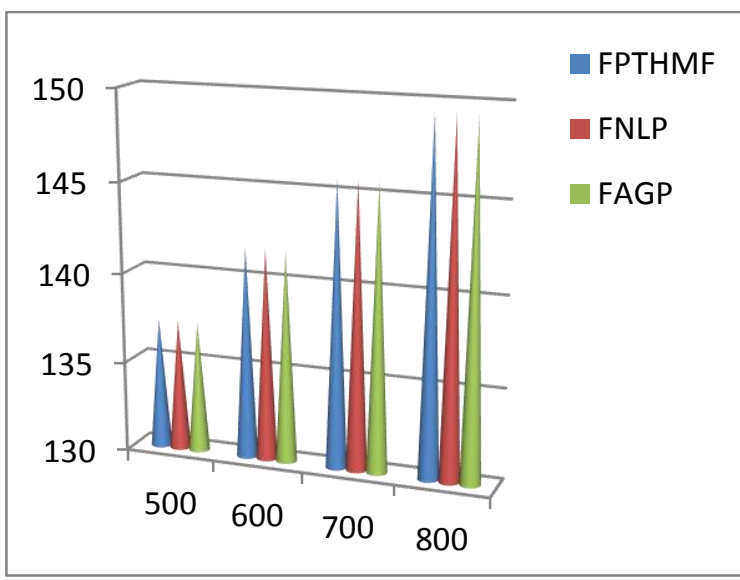

Figure 6: minimizing cost of $1^{\text {st }}$ item using different Methods for different values of $L_{1}$

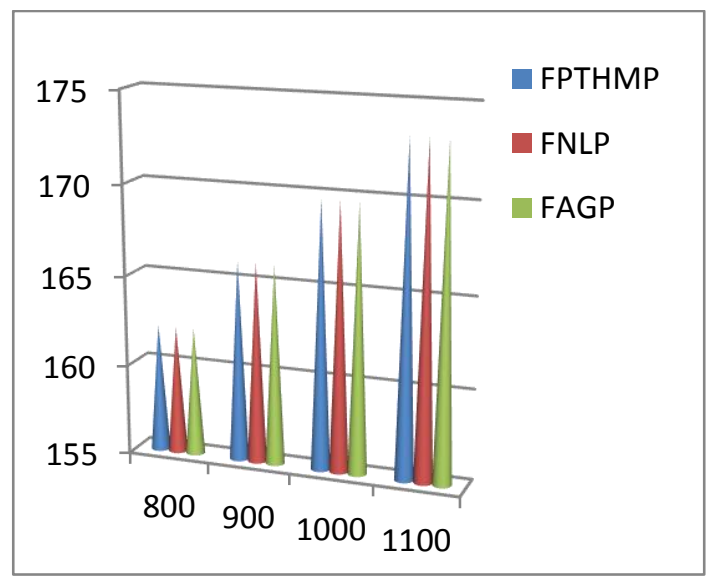

Figure 7: minimizing cost of $2^{\text {nd }}$ item using different Methods for different values of $L_{2}$

From the above figures $6 \& 7$ shows that minimum costs of the both items are increased when values of $L_{1}, L_{2}$ are increased and it is same for all methods.

Table 6: Optimal solution of MOSCIM by FPTHMF, FNLP and FAGP methods 
for different values of $\sigma_{1}, \sigma_{2}$.

\begin{tabular}{|l|c|c|c|c|c|c|c|c|c|c|}
\hline Method & $\sigma_{1}$ & $\sigma_{2}$ & $Q_{1}{ }^{*}$ & $P_{1}{ }^{*}$ & $D_{1}{ }^{*}$ & $T A C_{1}{ }^{*}$ & $Q_{2}{ }^{*}$ & $P_{2}{ }^{*}$ & $D_{2}{ }^{*}$ & $T_{A}{ }^{*}$ \\
\hline \multirow{5}{*}{ FPTHMF } & 0.5 & 0.6 & 16.44 & 28.51 & 1.41 & 137.23 & 22.07 & 33.57 & 1.44 & 162.08 \\
\cline { 2 - 11 } & 0.6 & 0.7 & 16.40 & 26.17 & 1.40 & 140.85 & 23.00 & 30.97 & 1.43 & 166.59 \\
\cline { 2 - 11 } & 0.7 & 0.8 & 16.29 & 23.86 & 1.40 & 144.14 & 21.91 & 28.77 & 1.42 & 170.58 \\
\cline { 2 - 11 } FNLP & 0.8 & 0.9 & 16.23 & 22.23 & 1.39 & 147.16 & 21.81 & 27.38 & 1.42 & 174.37 \\
\hline \multirow{5}{*}{ FAGP } & 0.5 & 0.6 & 16.33 & 28.51 & 1.41 & 137.23 & 22.07 & 33.57 & 1.44 & 162.08 \\
\cline { 2 - 11 } & 0.6 & 0.7 & 16.38 & 25.89 & 1.40 & 140.85 & 22.00 & 30.97 & 1.43 & 166.50 \\
\cline { 2 - 11 } & 0.7 & 0.8 & 16.29 & 23.86 & 1.40 & 144.14 & 21.86 & 28.91 & 1.43 & 170.58 \\
\cline { 2 - 11 } & 0.8 & 0.9 & 16.22 & 22.20 & 1.39 & 147.16 & 21.81 & 27.38 & 1.42 & 174.37 \\
\cline { 2 - 10 } & 0.5 & 0.6 & 16.34 & 28.39 & 1.41 & 137.23 & 22.07 & 33.57 & 1.44 & 162.08 \\
\cline { 2 - 10 } & 0.6 & 0.7 & 16.35 & 25.88 & 1.40 & 140.85 & 22.00 & 30.97 & 1.43 & 166.50 \\
\cline { 2 - 10 } & 0.7 & 0.8 & 16.28 & 23.86 & 1.40 & 144.14 & 21.85 & 28.91 & 1.43 & 170.58 \\
\cline { 2 - 10 } & 0.8 & 0.9 & 16.23 & 22.33 & 1.39 & 147.16 & 21.81 & 27.38 & 1.42 & 174.37 \\
\hline
\end{tabular}

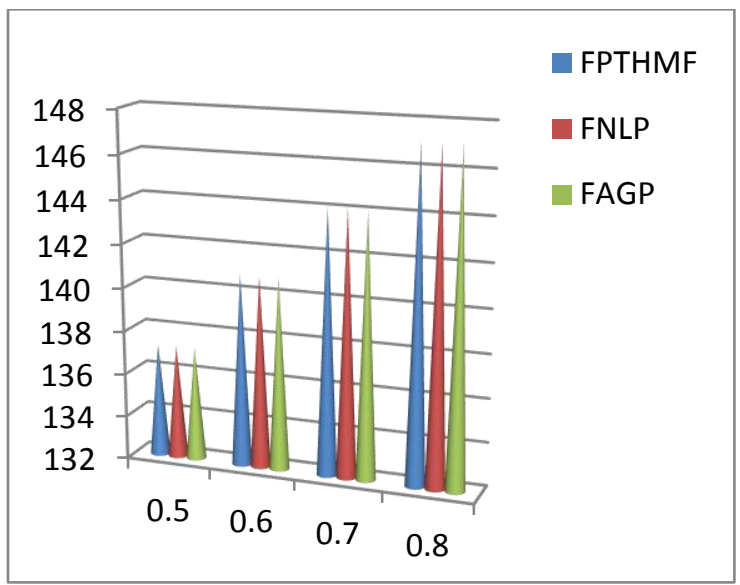

Figure 8: minimizing cost of $1^{\text {st }}$ item using different Methods for different values of $\sigma_{1}$

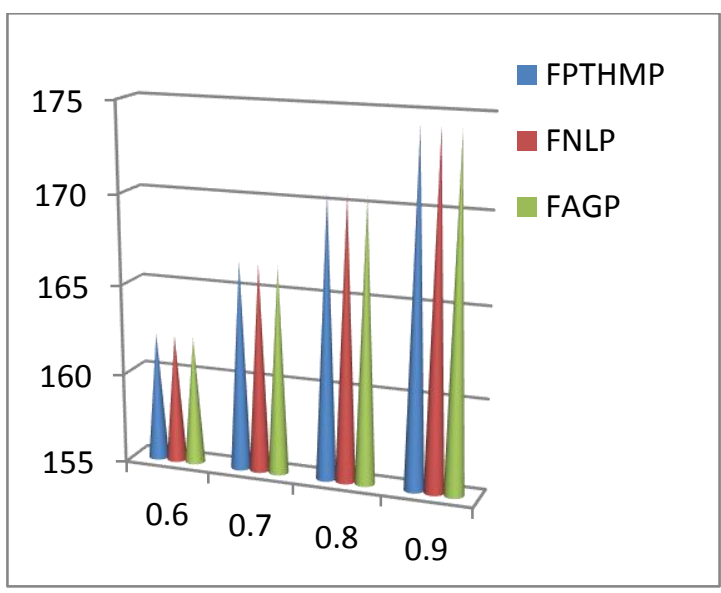

Figure 9: minimizing cost of $2^{\text {nd }}$ item using different Methods for different values of $\sigma_{2}$

From the above figures $8 \& 9$ shows that minimum costs of the both items are increased when values of $\sigma_{1}, \sigma_{2}$ are increased and it is same for all methods.

Table 7: Optimal solution of MOSCIM by FPTHMF, FNLP and FAGP methods for different values of $a_{1}, a_{2}$.

\begin{tabular}{|l|l|l|l|l|}
\hline Method & \multicolumn{1}{|c|}{$a_{1}$} & \multicolumn{1}{c|}{$a_{2}$} & \multicolumn{1}{|c|}{$T A C_{1}{ }^{*}$} & TAC ${ }^{*}$ \\
\hline \multirow{4}{*}{ FPTHMF } & 2 & \multicolumn{1}{c|}{2} & 137.72 & 169.01 \\
\cline { 2 - 6 } & 3 & 3 & 137.26 & 158.47 \\
\cline { 2 - 6 } & 4 & 4 & 137.23 & 158.42 \\
\cline { 2 - 6 } & 5 & 5 & 137.22 & 158.39 \\
\hline \multirow{4}{*}{ FNLP } & 2 & 2 & 137.84 & 168.97 \\
\cline { 2 - 6 } & 3 & 3 & 137.76 & 158.67 \\
\cline { 2 - 6 } & 4 & 4 & 137.45 & 158.53 \\
\hline
\end{tabular}




\begin{tabular}{|l|l|l|l|l|}
\hline & 5 & 5 & 137.23 & 158.42 \\
\hline \multirow{4}{*}{ FAGP } & 2 & 2 & 137.72 & 169.01 \\
\cline { 2 - 6 } & 3 & 3 & 137.61 & 158.89 \\
\cline { 2 - 6 } & 4 & 4 & 137.34 & 158.76 \\
\cline { 2 - 5 } & 5 & 5 & 137.23 & 158.39 \\
\hline
\end{tabular}

Table 8: Optimal solution of MOSCIM by FPTHMF, FNLP and FAGP methods for different values of $b_{1}, b_{2}$.

\begin{tabular}{|l|l|l|l|l|}
\hline Method & \multicolumn{1}{|c|}{$b_{1}$} & \multicolumn{1}{c|}{$b_{2}$} & $T_{A}{ }^{*}$ & $T^{*} C_{2}{ }^{*}$ \\
\hline \multirow{5}{*}{ FPTHMF } & 8 & 8 & 144.71 & 172.08 \\
\cline { 2 - 6 } & 9 & 9 & 140.56 & 166.52 \\
\cline { 2 - 6 } & 10 & 10 & 137.23 & 162.18 \\
\cline { 2 - 6 } & 11 & 11 & 134.49 & 162.08 \\
\hline \multirow{5}{*}{ FNLP } & 8 & 8 & 144.91 & 172.18 \\
\cline { 2 - 6 } & 9 & 9 & 140.65 & 166.59 \\
\cline { 2 - 6 } & 10 & 10 & 137.23 & 162.28 \\
\cline { 2 - 6 } & 11 & 11 & 134.43 & 162.08 \\
\hline \multirow{5}{*}{ FAGP } & 8 & 8 & 144.78 & 172.05 \\
\cline { 2 - 6 } & 9 & 9 & 140.66 & 166.52 \\
\cline { 2 - 6 } & 10 & 10 & 137.23 & 162.25 \\
\cline { 2 - 6 } & 11 & 11 & 134.49 & 162.08 \\
\hline
\end{tabular}

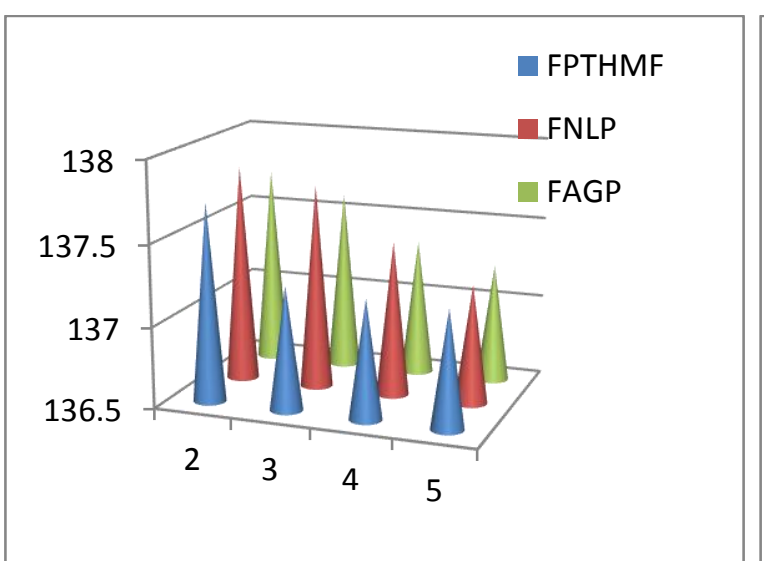

Figure 10: minimizing cost of $1^{\text {st }}$ item using different methods for different values of $a_{1}$

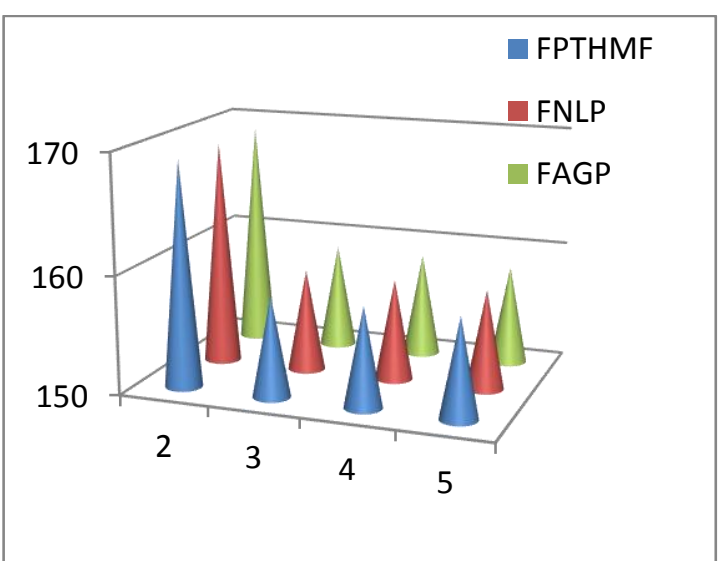

Figure 11: minimizing cost of $2^{\text {nd }}$ item using different methods for different values of $a_{2}$ 


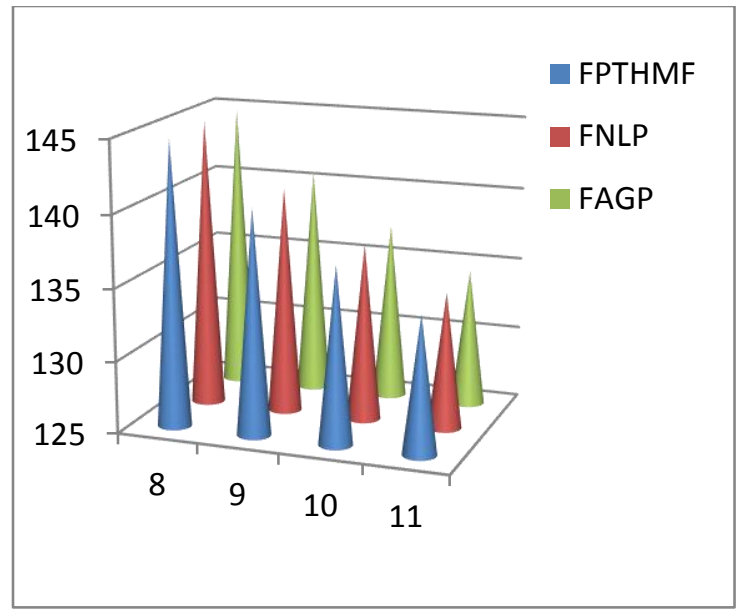

Figure 12: minimizing cost of $1^{\text {st }}$ item using different methods for different values of $b_{1}$

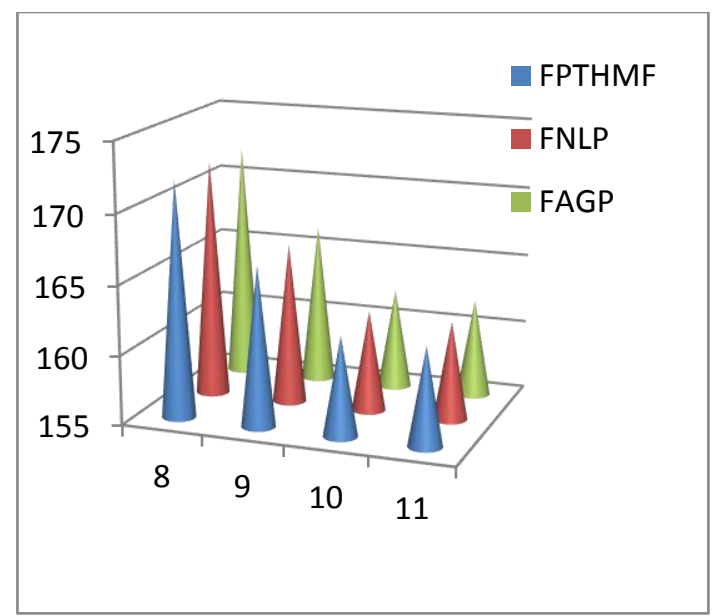

Figure 13: minimizing cost of $2^{\text {nd }}$ item using different methods for different values of $b_{2}$

From the above figures $10 \& 11$ shows that minimum costs of the both items are decreased when values of $a_{1}, a_{2}$ are increased and it is same for all methods. Also from figures $12 \& 13$ shows that minimum costs of the both items are decreased when values of $b_{1}, b_{2}$ are increased and it is same for all methods.

\section{Conclusions}

In this paper, purchase cost for the manufacturer is dependent on inventory lot size and production cost of the manufacturer is dependent on production rate. Also purchase cost of the retailer is dependent on demand rate of the customer. Multi item is considered in this model. The proposed MOSCIM has been solved by FPTHMF, FNLP, FAGP, WFNLP and WFAGP methods. LINGO13 software has been used to find the solution of the numerical example.

Any limitation is not considered in this paper. So in the future study, various limitations can be used such as on total storage area, ordering cost etc. Due to uncertainty, various types of fuzzy number like as triangular fuzzy number, trapezoidal fuzzy number, generalized trapezoidal fuzzy number etc. can used to form the fuzzy model in fuzzy sense.

\section{Acknowledgements:}

The authors are thankful to University of Kalyani for providing financial assistance through DST-PURSE (Phase-II) Programme. The authors are grateful to the reviewers for their comments and suggestions. 


\section{References}

1. Alfares, H. K., Ghaithan, A. M. (2016). Inventory and Pricing Model with Price Dependent Demand, Time-Varying Holding Cost, and Quantity Discounts. Computers \& Industrial Engineering, 94, 170-177.

2. Bhunia, A. K. and Shaikh, A. A. (2014). A deterministic inventory model for deteriorating items with selling price dependent demand and three-parameter Weibull distributed deterioration. International Journal of Industrial Engineering Computations, 5, 497-510.

3. Cárdenas-Barrón, L. E., Sana, S. S. (2015). Multi-item EOQ inventory model in a two layer supply chain while demand varies with promotional effort. Appl. Math. Mode, 39(21), 6725-6737.

4. Cardenas-Barron, L. E, Sana, S. S., (1014). A Production Inventory Model for a two echelon Supply Chain when demand is dependent on sales teams' initiatives. Int. J. Production Economics, 155, 249-258.

5. Goyal, S. K. (1976). An Integrated Inventory Model for a Single Supplier-Single Customer Problem. International Journal of Production research, 15(1), 107-111.

6. Ghosh, S. K., Sarkar, T. \& Chaudhuri, K. (2015). A Multi-Item Inventory Model for Deteriorating Items in Limited Storage Space with Stock-Dependent Demand. American Journal of Mathematical and Management Sciences, 34(2), 147-161.

7. Hui-Ming Wee, Chien-Chung Lo, Ping-Hui Hsu, (2009). A multi-objective joint replenishment inventory model of deteriorated items in a fuzzy environment. European Journal of Operational Research, 197, 620-631.

8. Kotb, A. M. Kotb, and Hala A. Fergancy (2011). Multi-item EOQ model with both demand depended unit cost and varying lead time via geometric programming. Applied Mathematics, 2, 551-555.

9. Mandal, W. A., Islam, S. (2016). Fuzzy Inventory Model for Deteriorating Items, with Time Depended Demand, Shortages, and Fully Backlogging. Pakistan Journal of Statistics and Operation Research. Vol. XII, No.1, pp101-109.

10. Pal, B., Sana, S.S., Chaudhuri, K. (2012). Three layer Supply Chain: A Production Inventory Model for re-workable Items. Applied Mathematics and Computation, 219, $530-543$. 
11. Pal, B., Sana, S.S., Chaudhuri, K. (2012). A Three Layer Multiple item Production Inventory Model for Multiple Suppliers and Retailers. Economic Modeling, 29, 27042710.

12. Pal, B., Sana, S. S., Chaudhuri, K. (2014). A Multiple Echelon Production Inventory system with Supply disruption. Journal of Manufacturing System, 33, 262-276.

13. Roy, T. K, Maiti, M (1998). Multi-Objective inventory models of deteriorating items with some constraints in a fuzzy environment, Computers Ops Res, 25(12), 10851095.

14. Rau, H., Wu, M. Y. and Wee, H. M. (2003). Integrated inventory model for deteriorating items under a multi-echelon supply chain environment. International Journal Production Economics, 86 (2), 155-168.

15. Sadeghi, J. (2015). A multi-item integrated inventory model with different replenishment frequencies of retailers in a two-echelon supply chain management: a tuned-parameters hybrid meta-heuristic, Opsearch, 52(4), 631-649.

16. Shah, N. H., Shah, B. J. and Wee, H. M. (2009). A lot size inventory model for the Weibull distributed deterioration rate with discounted selling price and stockdependent demand, Int. J. Data Analysis Techniques and Strategies, 1(4), 355-363.

17. Sridevi, G., Nirupama Devi, K. and Srinivasa Rao, K. (2010). Inventory model for deteriorating items with Weibull rate of replenishment and selling price dependent demand, Int. J. Operational Research, 9(3), 329-349.

18. Tzafestas, S., Kapsiotis, G. (1994). Coordinated control of manufacturing supply Chain System using multi-level Techniques. Computer Integrated Manufacturing System, 7, 206-212.

19. Yang, P. C. and Wee, H. M. (2000). Economic ordering policy of deteriorated item for vendor and buyer: an integrated approach. Production Planning and Control, 11 (3), 474-480.

20. Zhou, Y., Li, D. H. (2007). Coordinating order quantity decisions in the supply chain contract under random demand. Appl. Math. Model, 31, 1029-1038.

21. Zadeh, L. A. (1965). Fuzzy sets. Information and Control, 8, 338-353.

22. Zimmermann, H. J. (1985). Application of fuzzy set theory to mathematical programming, Information Science, 36, 29-58. 